\title{
14 Ceti: a probable Ap-star-descendant entering the Hertzsprung gap ${ }^{\star}$
}

\author{
M. Aurière ${ }^{1,2}$, R. Konstantinova-Antova ${ }^{3,1}$, P. Petit ${ }^{1,2}$, C. Charbonnel ${ }^{4,2}$, S. Van Eck ${ }^{5}$, J.-F. Donati ${ }^{1,2}$, \\ F. Lignières ${ }^{1,2}$, and T. Roudier ${ }^{1,2}$
}

1 Université de Toulouse, UPS-OMP, Institut de Recherche en Astrophysique et Planétologie, 31400 Toulouse, France e-mail: [michel.auriere; ppetit; jean-francois.donati; francois.lignieres; thierry.roudier] @irap.omp.eu

2 CNRS, Institut de Recherche en Astrophysique et Planétologie, 14 av. Edouard Belin, 31400 Toulouse, France

3 Institute of Astronomy and NAO, Bulgarian Academy of Sciences, 72 Tsarigradsko shose, 1784 Sofia, Bulgaria e-mail: renada@astro.bas.bg

${ }^{4}$ Geneva Observatory, University of Geneva, 51 Chemin des Maillettes, 1290 Versoix, Switzerland e-mail: corinne.charbonnel@unige.ch

5 Institut d'Astrophysique, Université libre de Bruxelles, Campus Plaine - CP 226, 1050 Bruxelles, Belgium e-mail: svaneck@ulb.ac.be

Received 1 April 2012 / Accepted 17 May 2012

\section{ABSTRACT}

\begin{abstract}
Context. 14 Ceti is a subgiant star of F spectral class that displays variations in the $S$-index of its Ca II $\mathrm{H} \& \mathrm{~K}$ lines and an X-ray emission that is stronger than the mean observed for its spectral class, which may be due to some magnetic activity.

Aims. We attempt to Zeeman-detect and study the magnetic field of 14 Ceti and to infer its origin.

Methods. We used the spectropolarimeter Narval at the Telescope Bernard Lyot, Pic du Midi Observatory, and the least squares deconvolution method to create high signal-to-noise ratio Stokes $V$ profiles. We derived the surface-averaged longitudinal magnetic field $B_{1}$. We also measured the $S$-index, and the radial velocity for each observation.

Results. 14 Ceti is Zeeman-detected for the 30 observed dates spanning from August 2007 to January 2012. The average longitudinal magnetic field does not reverse its sign, reaches about $-35 \mathrm{G}$, and shows some month-long-timescale variations in our 2008 and 2011-2012 observations. The $S$-index follows the same long-term trend as $B_{1} .14$ Ceti is confirmed as a single star without H-K emission cores. The strength of the observed surface magnetic field of 14 Ceti is one order of magnitude greater than the observed one for late $\mathrm{F}$ main-sequence stars, and is comparable to the values measured in the active late $\mathrm{F}$ pre-main-sequence star $\mathrm{HR} 1817$. On the other hand, taking into account the post-main-sequence evolution of an Ap star, an oblique rotator model can explain the strength of the magnetic field of 14 Ceti. The variations with a timescale of months observed for both the $B_{1}$ and $S$-index could be due to the rotation.
\end{abstract}

Conclusions. The most probable scenario to explain our observations appears to be that 14 Ceti is the descendant of a cool Ap star.

Key words. stars: individual: 14 Ceti - stars: magnetic field - stars: late-type

\section{Introduction}

14 Ceti (HD 3229, HR 143) has been classified as F5V (Gray 1989) and either F5IV or F5IV-V (Hoffleit \& Warren 1991). It was included as a "single star with no H-K emission" in a sample devoted to the "attempt to connect the rotations of mainsequence stars with their chromospheric properties" (Wilson 1966). It was observed over 25 years (1966-1991) as part of the HK Mount Wilson survey (Duncan et al. 1991; Baliunas et al. 1995). Baliunas et al. (1995) inferred a possible activity cycle of about five years, but with a poor confidence level. Noyes et al. (1984) predicted a rotational period of 2.4 days for 14 Ceti from the $\mathrm{Ca}$ II $\mathrm{H} \& \mathrm{~K}$ properties. 14 Ceti appears in the "ROSAT all-sky survey catalogue of optically bright main-sequence stars and subgiant stars" (Hünsch et al. 1998). Its X-ray luminosity of $0.33 \times 10^{30} \mathrm{erg} \mathrm{s}^{-1}$ is rather strong for an F5-type star (Schmitt et al. 1985), but is not unique in the ROSAT catalogue (Hünsch et al. 1998). Its $\left[L_{\mathrm{X}} / L_{\mathrm{bol}}\right]$ as presented by Bruevich et al. (2001) is also high.

* Based on data obtained using the Télescope Bernard Lyot at Observatoire du Pic du Midi, CNRS/INSU and Université de Toulouse, France.
Since the properties of its chromosphere and corona are indicative of magnetic activity, we included 14 Ceti in a magnetic (Zeeman) survey of evolved single intermediate-mass stars with Narval and detected its surface magnetic field (Aurière et al. 2009a). 14 Ceti then became the only star between F0 and F7 spectral types to be Zeeman-detected. It could then be either the hottest known star hosting a dynamo-driven surface magnetic field, or another example of a descendant of an Ap-star, such as EK Eri (e.g. Stepień et al. 1993; Aurière et al. 2011). We present here the Zeeman investigation that we have conducted to infer the origin of the magnetic activity of 14 Ceti, and discuss its possible evolutionary and magnetic status as inferred from stellar evolution models.

\section{Observations with Narval}

\subsection{Observations}

We used Narval at the TBL (2 m telescope Bernard Lyot at Observatoire du Pic du Midi, France), which is a copy of the new generation spectropolarimeter ESPaDOnS (Donati et al. 2006a). Narval consists of a Cassegrain polarimetric module connected by optical fibres to an echelle spectrometer. In polarimetric 
Table 1. Observations of 14 Ceti (for details, see Sect. 2.1).

\begin{tabular}{lccccc}
\hline \hline Date & $\begin{array}{c}\text { HJD } \\
\text { 2450 000+ }\end{array}$ & S-ind. & $\begin{array}{c}B_{1} \\
\mathrm{G}\end{array}$ & $\begin{array}{c}\sigma \\
\mathrm{G}\end{array}$ & $\begin{array}{c}R V \\
\mathrm{~km} \mathrm{~s}^{-1}\end{array}$ \\
\hline 18 Aug. 2007 & 4331.61 & 0.224 & -07.82 & 1.30 & 11.685 \\
14 Sep. 2008 & 4724.48 & 0.231 & -31.88 & 1.57 & 11.695 \\
16 Sep. 2008 & 4726.45 & 0.232 & -31.31 & 1.27 & 11.670 \\
19 Sep. 2008 & 4729.53 & 0.232 & -31.22 & 1.92 & 11.680 \\
20 Sep. 2008 & 4730.41 & 0.234 & -32.63 & 2.15 & 11.640 \\
25 Sep. 2008 & 4735.46 & 0.231 & -28.24 & 1.23 & 11.655 \\
21 Dec. 2008 & 4822.28 & 0.229 & -17.06 & 1.21 & 11.545 \\
22 Jul. 2011 & 5765.63 & 0.234 & -25.20 & 1.20 & 11.576 \\
27 Aug. 2011 & 5801.59 & 0.235 & -25.81 & 1.69 & 11.690 \\
24 Sep. 2011 & 5829.48 & 0.240 & -29.36 & 1.65 & 11.688 \\
27 Sep. 2011 & 5832.53 & 0.240 & -31.83 & 1.34 & 11.660 \\
01 Oct. 2011 & 5836.46 & 0.240 & -30.25 & 1.33 & 11.709 \\
04 Oct. 2011 & 5839.55 & 0.239 & -31.40 & 1.12 & 11.658 \\
10 Oct. 2011 & 5845.42 & 0.239 & -34.38 & 1.72 & 11.677 \\
12 Oct. 2011 & 5847.38 & 0.239 & -32.33 & 1.52 & 11.668 \\
14 Oct. 2011 & 5849.49 & 0.238 & -33.83 & 1.68 & 11.648 \\
16 Oct. 2011 & 5851.39 & 0.238 & -29.13 & 1.08 & 11.625 \\
25 Oct. 2011 & 5860.44 & 0.241 & -32.16 & 1.61 & 11.683 \\
30 Oct. 2011 & 5865.41 & 0.240 & -29.61 & 1.72 & 11.684 \\
08 Nov. 2011 & 5874.36 & 0.240 & -33.48 & 2.60 & 11.696 \\
12 Nov. 2011 & 5878.47 & 0.241 & -35.10 & 1.62 & 11.710 \\
16 Nov. 2011 & 5882.44 & 0.241 & -34.43 & 1.30 & 11.709 \\
21 Nov. 2011 & 5887.40 & 0.240 & -33.35 & 1.66 & 11.720 \\
27 Nov. 2011 & 5893.28 & 0.242 & -34.19 & 1.32 & 11.714 \\
08 Dec. 2011 & 5904.29 & 0.242 & -33.57 & 1.38 & 11.636 \\
12 Dec. 2011 & 5908.34 & 0.239 & -36.17 & 1.63 & 11.672 \\
08 Jan. 2012 & 5935.25 & 0.237 & -33.58 & 1.23 & 11.570 \\
19 Jan. 2012 & 5946.27 & 0.237 & -37.35 & 2.15 & 11.658 \\
22 Jan. 2012 & 5949.25 & 0.237 & -34.79 & 1.24 & 11.687 \\
26 Jan. 2012 & 5953.26 & 0.237 & -32.48 & 1.24 & 11.685 \\
\hline & & & & & \\
& & & & \\
\hline
\end{tabular}

mode, the instrument simultaneously acquires two orthogonally polarized spectra covering the spectral range from $370 \mathrm{~nm}$ to $1000 \mathrm{~nm}$ in a single exposure, with a resolving power of about 65000 .

A standard circular-polarization observation consists of a series of four sub-exposures between which the half-wave retarders (Fresnel rhombs) are rotated to exchange the paths of the orthogonally polarized beams within the whole instrument (and therefore the positions of the two spectra on the CCD), thereby reducing the spurious polarization signatures. The extraction of the spectra, including wavelength calibration, correction to the heliocentric frame, and continuum normalization, was performed using Libre-ESpRIT (Donati et al. 1997), a dedicated and automatic reduction package installed at TBL. The extracted spectra are produced in ASCII format, and consist of the normalised Stokes $I\left(I / I_{\mathrm{c}}\right)$ and Stokes $V\left(V / I_{\mathrm{c}}\right)$ parameters as a function of wavelength, along with their associated Stokes $V$ uncertainty $\sigma_{V}$ (where $I_{\mathrm{c}}$ represents the continuum intensity). We also include in the output "diagnostic null" spectra $N$, which are in principle featureless, and therefore serve to diagnose the presence of spurious contributions to the Stokes $V$ spectrum.

14 Ceti was observed on 7 nights between August 2007 and December 2008, then 23 nights during a survey in the 2011-2012 season. These observations are listed in Table 1. The exposure time for each observation was $40 \mathrm{~min}$ and the maximum signal-to-noise ratio $(\mathrm{S} / \mathrm{N})$ in Stokes $I$ per $2.6 \mathrm{~km} \mathrm{~s}^{-1}$ spectral bin was between 490 and 1000 .

To complete the Zeeman analysis, least squares deconvolution (LSD, Donati et al. 1997) was applied to all observations. This is a multi-line technique, similar to cross-correlation, which

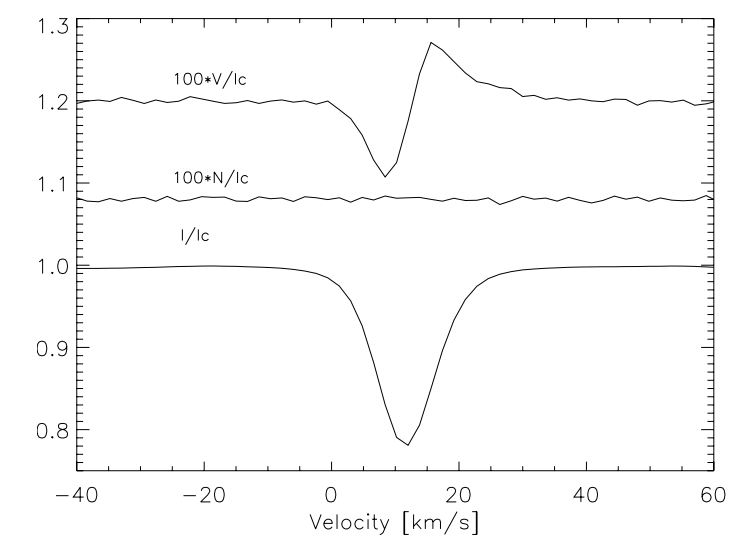

Fig. 1. Mean LSD profiles of 14 Ceti as observed with Narval on 25 Sep. 2008. Stokes $V$ (upper), $N$ nul polarization (middle), and Stokes I (lower) profiles are presented. For display purposes, the profiles are shifted vertically, and the Stokes $V$ as well as the $N$ profiles are expanded by a factor of 100 .

assumes that all spectral lines have the same profile, scaled by a certain factor. We used a digital mask with about 6500 photospheric lines, calculated for an effective temperature of $6500 \mathrm{~K}$, and $\log g=4$, using the Kurucz models (1993). In the present case, this method enabled us to derive Stokes $V$ profiles with a $\mathrm{S} / \mathrm{N}$ that was higher by a factor of about 30 than those for single lines. We then computed the longitudinal magnetic field $B_{1}$ in G, using the first-order moment method (Rees \& Semel 1979; Donati et al. 1997). Kochukhov et al. (2010) investigated the LSD method, showing that it provides estimates of the longitudinal magnetic field that are accurate to within a few percent for fields weaker than $1 \mathrm{kG}$, as which are considered here.

The activity of the star during the same nights was monitored by computing the $S$-index (defined from the Mount Wilson survey, Duncan et al. 1991) for the chromospheric Ca II H \& K line cores on our spectra. Our procedure was calibrated using the main-sequence solar-type stars of Wright et al. (2004).

We measured the radial velocity $(R V)$ of 14 Ceti on the LSD Stokes $I$ profiles using a Gaussian fit. The long-term stability of Narval is about $30 \mathrm{~m} \mathrm{~s}^{-1}$ (e.g. Moutou et al. 2007; Aurière et al. $2009 \mathrm{~b}$ ) and the absolute accuracy of individual measurements relative to the local standard of rest is about $1 \mathrm{~km} \mathrm{~s}^{-1}$.

Table 1 provides the date, HJD, the Ca II H\& K $S$-index, $B_{1}$ value and its uncertaintiy in $\mathrm{G}$, and $R V$.

\subsection{Magnetic field, S-index, and RV measurements}

The surface magnetic field of 14 Ceti is Zeeman-detected on each observation described in Table 1. Figure 1 shows the LSD Stokes $V, N$ null polarization, and Stokes $I$ profiles obtained on 25 September 2008. On each date, the Stokes $V$ profile does not display any peculiar details apart from the large-scale smooth behavior shown in Fig. 1. This can be explained by the small $v \sin i$ of $14 \mathrm{Cet}$, which is measured to be $5 \pm 1 \mathrm{~km} \mathrm{~s}^{-1}$ (de Medeiros \& Mayor 1999). Table 1 shows that the average longitudinal magnetic field does not reverse its sign and varies from about $-8 \mathrm{G}$ (17 August 2007) to about -30 G in September 2008 and during September 2011-January 2012.

Our observations confirm that 14 Ceti does not contain emission cores in the chromospheric Ca II H \& K lines (e.g. Fig. 2). In reality, the core profiles are as deep as the "wingnibs" described by Cowley et al. (2006) for main-sequence A-stars. The $S$-index values reported in Table 1 , which vary 


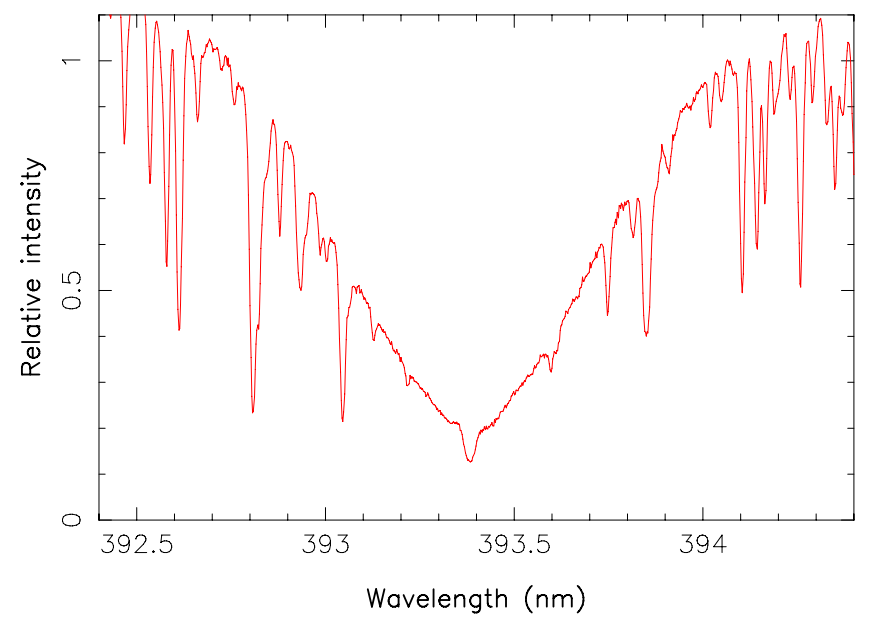

Fig. 2. Spectrum of 14 Ceti on 25 Sep. 2008 showing the core of the chromospheric $\mathrm{Ca}$ II $\mathrm{K}$ line.

between 0.224 and 0.242 , are consistent with those of the HK Mount Wilson survey (Duncan et al. 1991), which vary between 0.200 and 0.242 . The $S$-index values for 14 Ceti are constant during the September 2008 ( $S$-index $\approx 0.232$ ) and September-December 2011 observations $(S$-index $\approx 0.240$, see Fig. 3). The internal accuracy of the $S$-index is about 0.002 , as illustrated by the fluctuations observed in these phases of apparent constant magnetic activity of 2008 and 2011.

The $R V$ varies within the range $11.640-11.700 \mathrm{~km} \mathrm{~s}^{-1}$ during the four years of observations. It is weaker by $100 \mathrm{~km} \mathrm{~s}^{-1}$ on 21 December 2008, 22 July 2011 and 19 January 2012. These differences can be due to different atmospheric conditions (Moutou et al. 2007). The stability of $R V$ confirms that 14 Ceti is a single star.

\subsection{Magnetic field and S-index variations, and search for the rotational period}

Figure 3 plots the $30 B_{1}$ (upper graph) and $S$-index (lower graph) measurements. This figure shows that the large timescale variations in $B_{1}$ and $S$-index are the same. On 18 August 2007, the weakest values were observed for both measurements. In September-December 2008, a general decrease in $B_{1}$ and $S$-index was measured. In the July-December 2011 observations, there is a general increase, then a possible decrease during the January 2012 observations, in both the $B_{1}$ and $S$-index. If these variations were due to the rotational modulation, they would be indicative of a rather long rotational period, of a few months.

Since Noyes et al. (1984) predicted a short rotational period for 14 Ceti, we surveyed the magnetic field in September 2008 but did not observe significant variations for four observations spanning along six days. We performed a new survey over the 23 nights of the 2011-2012 observational season of 14 Ceti, obtaining a more finely sampled and longer series. Table 1 and Fig. 4 (upper graph) show some marginally significant variations in $B_{1}$ during the September-January span of the 2011-2012 observations with a timescale of a few tens of days and an amplitude of about 3-4 G. Figure 4 (lower graph) shows that the $S$-index does not correlate with $B_{1}$ on this timescale. We then searched for periodic variations using the procedure described in Petit et al. (2002) and the Zeeman Doppler imaging models of Donati et al. (2006b), in a way described in Aurière et al. (2011). We could not determine any significant period in the
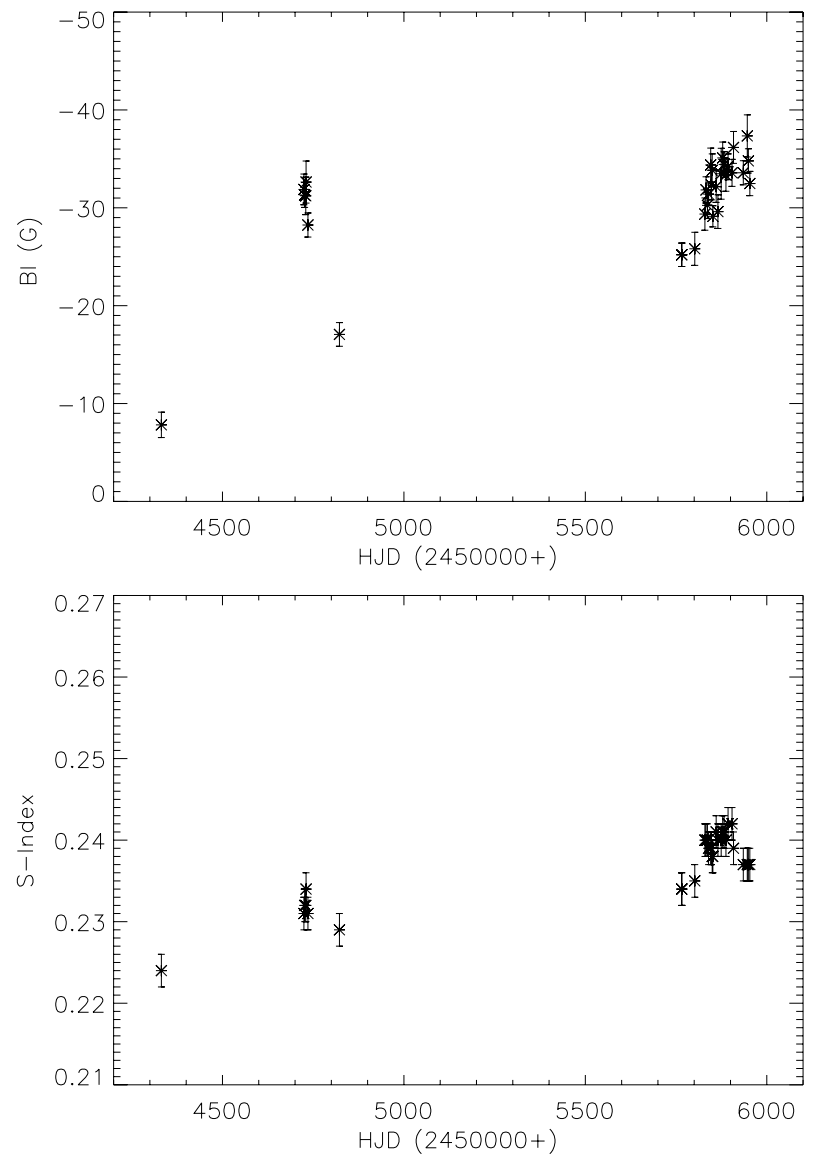

Fig. 3. Variations in the average longitudinal magnetic field and the $S$-index of 14 Ceti with the Julian date for the full 2007-2012 span of observations. The errors in $B_{1}$ are taken from Table 1, and for the $S$-index an error of 0.002 is illustrated.

range 1-100 days, and the smallest reduced $\chi^{2}$ was about 6 . This shows that the small timescale variations in $B_{1}$ seen in Fig. 4 for September 2011-January 2012 are not due to the rotation of the star, but are dominated by the errors in $B_{1}$ and possible intrinsic magnetic variations.

In summary, after investigating our complete data set, we were unable to detect a rotational period shorter than 100 days, but the observed variations in both the $B_{1}$ and $S$-index might correspond to a rotational period of a few months.

\section{Abundances, fundamental parameters, and evolutionary status of 14 Ceti}

Van Eck et al. (in prep.) determined the stellar parameters of 14 Ceti and measured the abundances of several elements based on a detailed synthesis analysis of some of the spectra of Narval listed in Table 1. Some results of interest are already discussed in the present paper. In particular, the derived effective temperature of $T_{\text {eff }}=6425 \pm 25 \mathrm{~K}$ can be used to pinpoint the position of 14 Ceti in the Hertzsprung Russell diagram as shown in Fig. 5, where the luminosity of the star was obtained using the Hipparcos parallax of van Leeuwen (2007), the $V$ magnitude from the 1997 Hipparcos catalogue, and the bolometric correction following Flower $(1996 ; \mathrm{BC}=0)$. When compared to the standard evolutionary tracks of Charbonnel \& Lagarde (2010) at solar metallicity that corresponds to the value of $[\mathrm{Fe} / \mathrm{H}] \mathrm{de}-$ rived from the Narval spectra, a mass of $1.5 M_{\odot}$ was inferred for 14 Ceti. As can be seen in Fig. 5, the star is expected to 

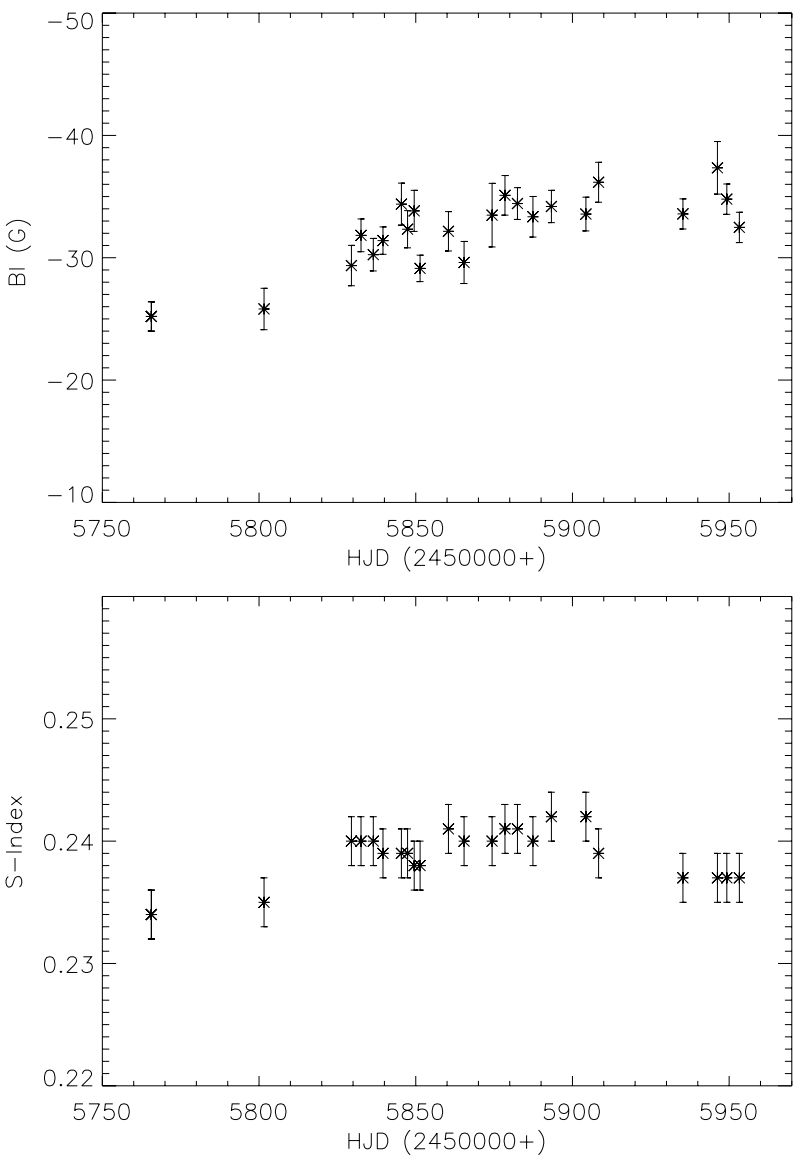

Fig. 4. Variations in the average longitudinal magnetic field and the $S$-index of 14 Ceti with the Julian date during the 2011-2012 season. The errors in $B_{1}$ are taken from Table 1 , and for the $S$-index an error of 0.002 is illustrated.

have entered the Hertzsprung gap. As previously mentioned, 14 Ceti has abundances close to solar, except for $\mathrm{Li}$ and $\mathrm{Be}$, which are depleted as previously found by Lèbre et al. (1999) and Boesgaard et al. (2001). As discussed in more detail in Van Eck et al. (in prep.), the $\mathrm{Li}$ and Be observed values can be explained by rotation-induced mixing for two different assumptions, i.e., considering that the star was either a fast rotator that has undergone no magnetic braking while on the main-sequence, or that it was initially a slow rotator that has undergone strong magnetic braking.

Since the value of $v \sin i$ is relevant to the present work, we made our own check for its determination. Feltzing et al. (2007) derived a macroturbulence $v_{\mathrm{RT}}=5.44 \mathrm{~km} \mathrm{~s}^{-1}$ for 14 Ceti. This value is smaller than the average macroturbulence measured for F5 IV stars (e.g. Gray 1992). Feltzing et al. (2007) found that adding a rotational broadening did not improve the spectral line fitting. Adopting a macroturbulence of $v_{\mathrm{RT}}=5.44 \mathrm{~km} \mathrm{~s}^{-1}$ and given the spectral resolution of Narval, we verified that a value of $v \sin i$ larger than $4 \mathrm{~km} \mathrm{~s}^{-1}$ can be excluded.

\section{Origin of the magnetic field of 14 Ceti}

14 Ceti is the only Zeeman-detected low-mass star in the range between F0 (the coolest Ap stars, whose magnetic fields are large-scale and assumed to be of fossil origin; Johnson et al. 2006) and F7 (the hottest stars Zeeman-detected, whose magnetic fields are believed to be of dynamo origin; Donati \& Landstreet 2009).

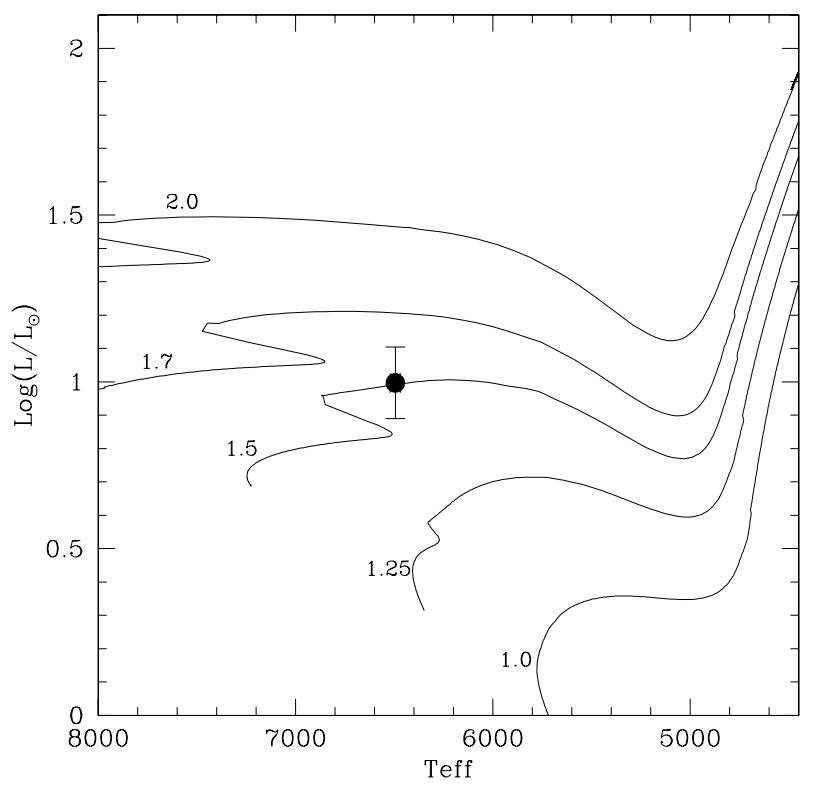

Fig. 5. Position of 14 Ceti in the Hertzsprung Russell diagram, using the effective temperature derived by Van Eck et al. (in prep.) and the Hipparcos luminosity (see text). Standard evolutionary tracks at solar metallicity by Charbonnel \& Lagarde (2010) are shown from the zeroage main-sequence on, for different masses as indicated by the labels.

The detected surface magnetic field of 14 Ceti, with a longitudinal magnetic field currently reaching $-30 \mathrm{G}$, is strong for an evolved star (e.g. Konstantinova-Antova 2009; Aurière et al. 2009a, and in prep.). This value is one order of magnitude greater than the observed one at the surface of a few of the hottest main-sequence F7 stars that have been previously Zeeman-detected (mean field of a few G, e.g. Donati \& Landstreet 2009), and reaches a value observed in the highly active young F7-F9 star HR 1817 (mean field of 25-50 G, Marsden 2006, and priv. comm.). On the other hand, the magnetic strength of 14 Ceti is moderate, compared to those observed for the cool Ap stars, which are generally greater than a few hundred $G$ (e.g. Ryabchikova et al. 2005). One has to take into account the evolution of the star, since 14 Ceti is entering the Hertzsprung gap, where the convective envelope starts deepening (which is favourable for a dynamo-driven magnetic field), and when the stellar radius increases (which would weaken a large-scale fossil magnetic field).

The two natural ways of explaining the magnetic strength of 14 Ceti are then either the star is a very fast rotator with a dynamo-driven magnetic field, or it is the descendant of a cool Ap star, which is a rather slow rotator (Abt \& Morrell 1985). The determination of the rotational period of 14 Ceti would have enabled us to infer directly the origin of its surface magnetic field, but we did not succeed in our investigation (Sect. 2.2), though a period longer than a few months may be present. In the following, we discuss the two different options and their implications.

\subsection{Ceti as a fast rotator}

At the location of 14 Ceti in the HRD, we note that the $1.5 M_{\odot}$, $Z_{\odot}$, rotating model of Charbonnel \& Lagarde (2010), which was computed with an initial velocity of $150 \mathrm{~km} \mathrm{~s}^{-1}$ and presented by Van Eck et al. (in prep.), has a radius of $2.51 R_{\odot}$, a surface velocity of $75 \mathrm{~km} \mathrm{~s}^{-1}$, and fits the observed $\mathrm{Li}$ and Be abundances. From these parameters and assuming solid-body rotation, we infer a rotational period of about $1.7 \mathrm{~d}$. In these 
conditions, the inclination should be less than $4^{\circ}$ to explain the observed $v \sin i\left(\leq 4 \mathrm{~km} \mathrm{~s}^{-1}\right.$, see Sect. 3$)$. The model also gives a convective turnover time of about four days at the bottom of the convective envelope. The inferred Rossby number at the bottom of the convective enveloppe is therefore about 0.5 and a solartype dynamo could be driven (Durney \& Latour 1978) at this evolutionary stage for a fast rotating star. However, at the location of 14 Ceti in the HRD, the theoretical external convection zone is thin (about $8 \%$ of the stellar radius) and its mass corresponds to that of a main-sequence star of about $1.25 M_{\odot}$, i.e. a late FV star (using models of Charbonnel \& Lagarde 2010). The magnetic field strength of 14 Ceti would be expected to be comparable to those that are Zeeman-detected among the most active late $\mathrm{F}$ main-sequence stars.

For the hottest stars on the main-sequence where a magnetic field is believed to be dynamo-driven, a survey of solartype stars is in progress with Narval (e.g. Petit et al. 2008). The hottest star Zeeman-detected up to now by this survey is the F7V star HD 75332, as shown in Fig. 3 of Donati \& Landstreet (2009), and the surface magnetic field is of a few G. Other late $F$ stars have also been Zeeman studied in the case of planethosting stars. The first of these was $\tau$ Boo $\left(\mathrm{F} 7 \mathrm{~V}, P_{\text {rot }}=3.1 \mathrm{~d}\right)$ for which the mean magnetic field strength is measured to be a few G (Catala et al. 2007; Donati et al. 2008; Fares et al. 2009). Several F stars observed by CoRoT also deserved deep Zeeman investigations with ESPaDOnS and Narval. The active stars HD 49933 (Garcia et al. 2010; Ryabchikova et al. 2009) and WASP-12 (Fossati et al. 2010) were deeply observed but have no Zeeman detection. In the course of the present study, we also performed an observation with Narval of a kind of twin of 14 Ceti with the same spectral class and the same X-ray emission (HD 25621, Hünsch et al. 1998): we achieved no Zeeman detection, with a $1 \sigma$ upper limit for $B_{1}$ of $2 \mathrm{G}$. From these surveys, only very few late $\mathrm{F}$ stars have been Zeeman-detected, and the magnetic field of 14 Ceti is in general one order of magnitude stronger than theirs. Only the very active F7-9 young star HR 1817 (Gagné et al. 1999; Budding et al. 2002) has a magnetic field that has been measured to be strong as that of 14 Ceti (HR 1817: $P_{\text {rot }} \approx 1 \mathrm{~d}, B_{\text {mean }} \approx 25-50 \mathrm{G}$, Mardsden et al. 2006, 2010; Marsden, priv. comm.). $B_{\text {mean }}$ is the mean unsigned magnetic field and is in general stronger than $\left|B_{1}\right|^{\max }$ (e.g. $\xi$ Boo A, Morgenthaler et al. 2012). However, HR 1817 has the same characteristics as very active stars, including strong $\mathrm{Ca}$ II $\mathrm{H} \& \mathrm{~K}$ emission cores and X-ray emission at the $10^{30} \mathrm{erg} \mathrm{s}^{-1}$ level, in contrast to 14 Ceti, which has a more quiet chromosphere and not so strong coronal activity. As discussed previously, the convective envelope of $14 \mathrm{Ceti}\left(T_{\text {eff }}=6425 \mathrm{~K}\right)$ is relatively small and associated with a theoretical convective turnover-time of about four days (Van Eck et al., in prep.), when it is expected to be about $20 \mathrm{~d}$ for HR 1817 (Gagné et al. 1999, $T_{\text {eff }}=6100 \mathrm{~K}$, Marsden, priv. comm.), which is more favourable for dynamo operation.

\subsection{Ceti as the descendant of a cool Ap star}

Owing to its small $v \sin i\left(\leq 4 \mathrm{~km} \mathrm{~s}^{-1}\right.$ see Sect. 3), and strong $\left|B_{1}\right|, 14$ Ceti is a natural candidate for an Ap star descendant. Assuming solid rotation, the rotational period (in day) is longer than or equal to:

$P_{\text {rot }} \geq \frac{50.6 R_{*} \sin i}{4}$

where $R_{*}$ is the radius of the star in $R_{\odot}$ (see e.g. Aurière et al. 2007). For a representative value of $60^{\circ}$ for $i$ and using the star radius inferred from the model of Charbonnel \& Lagarde (2010), we find a rotational period longer than 27 days.

For an Ap-star descendant, assuming conservation of magnetic flux during the post-main-sequence evolution (Stepien 1993), the surface magnetic field strength is expected to decrease as the stellar radius expands as $\left(R_{*}\right)^{-2}$. On the main-sequence, the $1.5 M_{\odot}$ progenitor of 14 Ceti, would be of F2 spectral type (Allen 2000) with a radius of $1.4 R_{\odot}$. The present magnetic strength of 14 Ceti would therefore be 3.2 times weaker than that of its Ap star progenitor. If we consider that the topology of the magnetic field can be still be represented as a dipole (as in the case of the Ap-star-descendant EK Eri, which is more evolved than 14 Ceti, Aurière et al. 2011), its $B_{1}$ variations could be fitted by an oblique rotator model (ORM, Stibbs 1950), similar to that presented by Preston (1967) and reviewed by Aurière et al. (2007). In this framework and for any geometry, the strength of the dipole is greater than

$B_{\mathrm{d}} \geq 3.3 B_{\ell}^{\max }$.

In our case, $B_{\mathrm{d}}$ is greater than $120 \mathrm{G}$, and the dipole strength of the Ap star progenitor is greater than about $370 \mathrm{G}$, i.e. stronger than the minimum magnetic field for an Ap-star of about $300 \mathrm{G}$ found by Aurière et al. (2007). The observed values of $B_{\mathrm{d}}$ for cool Ap stars encompass the whole strength range of magnetic fields for Ap stars (e.g. Aurière et al. 2007; studied EP UMa, an F0sp star, for which a dipole weaker than $500 \mathrm{G}$ is inferred; for HD 101065, F0p, Cowley et al. 2000, derived a magnetic field strength of $2.3 \mathrm{kG}$ ). Since the $B_{1}$ of 14 Ceti does not reverse its sign, the possible ORM have limited geometries, but enable us to consider a large span of dipole strengths typical of an Ap star on the main-sequence, to explain the observed strength of about $35 \mathrm{G}$ observed for $\left|B_{1}\right|^{\max }$. The search for the rotational period of 14 Ceti presented in Sect. 2.3 suggests that it could be about several months. A period $P_{\text {rot }}$ of 4 months, for example, would imply that $v \sin i$ is smaller than 1 , which is compatible with our investigation (Sect. 3).

We note that the solar abundances reported by Van Eck et al. (in prep.) for 14 Ceti, including elements that are hugely overabundant in Ap stars, might appear surprising for an Ap-stardescendant that is just leaving the main-sequence. However, the atmospheric-abundance anomalies induced in main-sequence Ap stars by gravitational and radiatively driven atomic diffusion are expected to be erased by the deepening convective envelope at the location of 14 Ceti in the HRD for a $1.5 M_{\odot}, Z_{\odot}$ star (Vick $\&$ Richard, priv. comm.). This was confirmed by the rotating models presented by Van Eck et al. for a $1.5 M_{\odot}, Z_{\odot}$ star computed with slow initial velocity between $20 \mathrm{~km} \mathrm{~s}^{-1}$ and $50 \mathrm{~km} \mathrm{~s}^{-1}$ and assuming magnetic braking, as required to explain the low $\mathrm{Li}$ and $\mathrm{Be}$ abundances of 14 Ceti. In this case, the increase in both shear and meridional circulation induced by the magnetic braking in the presence of an existing fossil magnetic field is expected to wipe out the Ap overabundances.

\section{Conclusion}

14 Ceti is a rare star that has been Zeeman-detected in the temperature range where both fossil Ap-type and dynamo-driven magnetic fields could coexist. Evolutionary models for both of these hypotheses (corresponding to slow and fast rotators, respectively) can explain the observed abundances of 14 Ceti (Van Eck et al., in prep.). The observed strong $\left|B_{1}\right|$ for 14 Ceti is unexpected for a dynamo-driven magnetic field in an F-type star on the main-sequence or beyond (Sect. 4.1), and is only 
approached by the very active F7-F9 young star HR 1817. On the other hand, finding that $\left|B_{1}\right|$ is about $30 \mathrm{G}$ for an Ap-stardescendant in the evolutionary state of 14 Ceti is more natural since it would correspond to the dipole strength in the range observed on the main-sequence for cool Ap stars, using an ORM of reasonable geometry and assuming conservation of the magnetic flux during the evolution. We have been unable to determine the rotational period of 14 Ceti. However, investigating our measurements obtained in 2011-2012, we could not detect any period shorter than 100 days, whereas when we consider our complete data set, the observed variations of both the $B_{1}$ and $S$-index suggest a rotational period of a few months.

If 14 Ceti is an evolved Ap star as we presently propose, its magnetic properties can be naturally explained, as in the case of EK Eri (Aurière et al. 2008, 2011), by the interplay of convection with a dipolar magnetic field of fossil origin. That 14 Ceti abundances are solar and that Ap star overabundances were wiped out on its entering the Hertzsprung gap (Van Eck et al., in prep.), explains why no evolved Ap stars have so far been detected by mean of spectroscopy.

Acknowledgements. We thank the TBL team for providing service observing with Narval and the PNPS of CNRS/INSU for financial support. We acknowledge the use of the CNRS/INSU CDAB datacenter operated by the Université Paul Sabatier, Toulouse-OMP (Tarbes, France; http://tblegacy . bagn.obs-mip.fr). The observations obtained in 2008 were funded under an OPTICON grant. R. K.-A. acknowledges the possibility of working for six months in 2010 as a visiting researcher in LATT, Tarbes, under Bulgarian NSF grant DSAB 02/3/2010. She also acknowledges partial support under the Bulgarian NSF contract DO 02-85 and contract RILA 01/14. C.C. acknowledges financial support from the Swiss National Science Foundation (FNS).

\section{References}

Abt, H. A., \& Morrell, N. I. 1995, ApJS, 99, 135

Allen, C. W. 2000, Astrophysical quantities, ed. A. N. Cox (AIP Press)

Aurière, M., Wade, G. W., Silvester, J., et al. 2007, A\&A, 475, 1053

Aurière, M., Konstantinova-Antova, R., Petit, P., et al. 2008, A\&A, 499, 491

Aurière, M., Konstantinova-Antova, R., \& Petit, P. 2009a, in Cosmic Magnetic Fields: From Planets, to Stars and Galaxies, eds. K. G. Strassmeier, A. G. Kosovichev, \& J. E. Beckman (Cambridge: Cambridge University Press), 431 Aurière, M., Wade, G. A., Konstantinova-Antova, R., et al. 2009b, A\&A, 504, 231

Aurière, M., Konstantinova-Antova, R., Petit, P., et al. 2011, A\&A, 534, A139

Baliunas, S. L., Donahue, R. A., Soon, W. H., et al. 1995, ApJ, 438, 269

Boesgaard, A. M., Deliyannis, C. P., King, J. R., \& Stephens, A. 2001, ApJ, 553, 754

Budding, E., Carter, B. D., Mengel, M. W., Slee, O. B., \& Donati, J.-F. 2002, PASA, 19, 527

Bruevich, E. A., Katsova, M. M., \& Sokolov, D. D. 2001, Astron. Rep., 45, 718
Catala, C., Donati, J.-F., Shkolnik, B. D., \& Alecian, E. 2007, MNRAS, 374, L42

Charbonnel, C., \& Lagarde, N. 2010, A\&A, 522, A10

Cowley, C. R., Ryabchikova, T., Kupka, F., et al. 2000, MNRAS, 317, 209

Cowley, C. R., Hubrig, S., \& Kamp, I. 2006, ApJS, 163, 393

De Medeiros, J. R., \& Mayor, M. 1999, A\&AS, 139, 433

Donati, J.-F., \& Landstreet, J. D. 2009, ARA\&A, 47, 333

Donati, J.-F., Semel, M., Carter, B. D., et al. 1997, MNRAS, 291, 658

Donati, J.-F., Catala, C., Landstreet, J., \& Petit, P. 2006a, in Solar Polarization Workshop n4, eds. R. Casini, \& B. Lites (San Francisco: ASP), ASPC Ser., 362,358

Donati, J.-F., Howarth, I. D., Jardine, M. M., et al. 2006b, MNRAS, 370, 629

Donati, J.-F., Moutou, C., Farès, R., et al. 2008, MNRAS, 385, 1179

Duncan, D. K., Vaughan, A. H., Wilson, O. C., et al. 1991, ApJS, 76, 383

Durney, B. R., \& Latour, J. 1978, GApFD, 9, 241

Feltzing, S., Fohlman, M., \& Bensby, T. 2007, A\&A, 467, 665

Flower, P. J. 1996, ApJ, 469, 355

Fossati, L., Bagnulo, S., Elmasli, A., et al. 2010, ApJ, 720, 872

Gagné, M., Valenti, J. A., Linsky, J. L., et al. 1999, ApJ, 515, 423

Gray, R. O. 1989, AJ, 98, 1049

Gray, D. F. 1992, in The Observation and Analysis of Stellar Photospheres, Camb. Astrophys. Ser., 20

Hoffleit, D., \& Warren, W. 1991, in Astronomical Data Center CD ROM, Selected Astronomical Catalogs, 1, eds. L. E. Brotzman, \& S. E. Gessmer, 5 th edn.

Hünsch, M., Schmitt, J. H. M. M., \& Voges, W. 1998, A\&AS, 132, 155

Johnson, N., Wade, G. A., Allen, A., et al. 2006, in Solar Polarization 4, eds. R. Casini, \& B. W. Lites (San Francisco: ASP), ASP Conf. Ser., 358, 393

Kochukhov, O., Makaganiuk, V., \& Piskunov, N. 2010, A\&A, 524, A5

Konstantinova-Antova, R., Aurière, M., Schröder, K.-P., \& Petit, P. 2009, in Cosmic Magnetic Fields: From Planets, to Stars and Galaxies, eds. K. G. Strassmeier, A. G. Kosovichev, \& J. E. Beckman (Cambridge: Cambridge University Press), 433

Kurucz, R. L. 1993, SAO, Cambridge, CDROM 18

Lèbre, A., de Laverny, P., de Medeiros, J. R., Charbonnel, C., \& da Silva, L. 1999, A\&A, 345, 936

Marsden, S. C., Mengel, M. W., Donati, J.-F., et al. 2006, in Solar Polarization 4 (San Francisco: ASP), ASP Conf. Ser., 358, 401

Marsden, S. C., Jeffers, S. V., Donati, J.-F., et al. 2010, in Solar and Stellar Variability: Impact on Earth and Planets, eds. A. G. Kovichev, A. H. Andrei, \& J.-P. Rozelot (Cambridge: Cambridge University Press), Proc. IAU Symp., 264, 130

Morgenthaler, A., Petit, P., Saar, S., et al. 2012, A\&A, 540, A138

Moutou, C., Donati, J.-F., Savalle, R., et al. 2007, A\&A, 473, 651

Noyes, R. W., Hartmann, L. W., Baliunas, S. L., Duncan, D. K., \& Vaughan, A. H. 1984, ApJ, 279, 763

Petit, P., Donati, J.-F., \& Collier Cameron, A. 2002, MNRAS, 334, 374

Petit, P., Dintrans, B., Solanki, S. K., et al. 2008, MNRAS, 388, 80

Preston, G. W. 1967, ApJ, 150, 547

Rees, D. E., \& Semel, M. 1979, A\&A, 74, 1

Ryabchikova, T. A., Wade, G. A., Aurière, M., et al. 2005, A\&A, 429, 55

Ryabchikova, T., Fossati, L., \& Shulyak, D. 2009, A\&A, 506, 203

Schmitt, J. H. M. M., Golub, L., Harnden, Jr. F. R., et al. 1985, ApJ, 290, 307

Stepień, K. 1993, ApJ, 416, 368

Stibbs, D. W. N. 1950, MNRAS, 110, 395

van Leeuwen, F. 2007, A\&A, 474, 653

Wilson, O. C. 1966, ApJ, 144, 695

Wright, J. T., Marcy, G. W., Butler, R. P., \& Vogt, S. S. 2004, ApJS, 152, 261 